\title{
EVALUACIÓN DE OCRATOXINA A EN Theobroma cacao L. “CACAO BLANCO” DURANTE EL PROCESO DE COSECHA, FERMENTADO, SECADO Y ALMACENADO
}

\author{
Norma Ramos ${ }^{* a}$, Américo Castro $^{\mathrm{a}}$, José Juárez ${ }^{\mathrm{a}}$, Otilia Acha de la Cruz ${ }^{\mathrm{b}}$, Nadia Rodríguez \\ Jesús Blancas ${ }^{\mathrm{b}}$, Jackeline Escudero ${ }^{\mathrm{a}}$, Arnaldo Navarro ${ }^{\mathrm{a}}$.
}

\section{RESUMEN}

Se analizó la presencia de la ocratoxina A (OTA), una micotoxina con propiedades carcinogénicas, en el cacao blanco de Piura. Las muestras fueron colectadas en cuatro etapas de la producción de granos: cosecha, fermentado, secado y almacenado. El análisis se hizo tanto en la cáscara del grano como en el cotiledón. Las muestras fueron colectadas del centro de acopio de la Asociación de Pequeños Productores de Cacao de Piura (APPROCAP). La OTA se extrajo de las muestras utilizando bicarbonato de sodio $1 \%$, se purificó mediante columnas de inmunoafinidad y su posterior cuantificación por cromatografía líquida de alto rendimiento (CLAR). En la etapa del fermentado se encontró una concentración detectable de OTA $(1,92 \pm 0,02 \mu \mathrm{g} / \mathrm{kg})$. Las etapas de cosecha, secado y almacenado en el proceso de producción de los granos de cacao no manifestaron presencia de OTA.

Palabras clave: Ocratoxina A (OTA), cacao blanco de Piura, columnas de inmunoafinidad, cromatografía líquida de alto rendimiento.

\section{OCHRATOXIN A EVALUATION IN Theobroma cacao L. "WHITE COCOA" DURING THE HARVESTING PROCESS, FERMENTED, DRIED AND STORED}

\begin{abstract}
The presence of ochratoxin A(OTA), a mycotoxin with carcinogenic properties, was analyzed in the white cocoa of Piura. Samples were collected in four stages of grain production: harvest, fermented, dried and stored. The analysis was done both on the grain shell and on the cotyledon. The samples were collected from the collection center of the Association of Small Producers of Cocoa Piura (APPROCAP). OTA was extracted from the samples using $1 \%$ sodium bicarbonate, purified by immunoaffinity columns and then quantified by high performance liquid chromatography (HPLC). A detectable concentration of OTA (1.92 \pm $0.02 \mu \mathrm{g} / \mathrm{kg}$ ) was found in the fermentation stage. The harvest, drying and storage stages in the cocoa grain production process did not show OTA.
\end{abstract}

Key words: Ochratoxin A (OTA), Cacao White Piura, immunoaffinity columns, High Performance Liquid Chromatography (HPLC)

\footnotetext{
a Facultad de Farmacia y Bioquímica, Universidad Nacional Mayor de San Marcos, Jr. Puno 1002. Jardín Botánico. Lima-Perú. nozjuli@gmail.com

b Facultad de Ingeniería Química y Textil, Universidad Nacional de Ingeniería, Av. Túpac Amaru 210, Lima 25, Perú.
} 


\section{INTRODUCCIÓN}

La ocratoxina A (OTA) es una toxina generada por hongos, especialmente del género Aspergillus y Penicillium, con propiedades nefrotóxicas, inmunotóxicas, teratogénicas y carcinogénicas; que ataca diversos cultivos, entre ellos el cacao ${ }^{1}$.

El método ampliamente utilizado para el análisis de OTA, en diversas matrices, es la cromatografía líquida de alto rendimiento con detector de fluorescencia debido a su sensibilidad para detectar concentraciones inferiores a $1 \mu \mathrm{g} / \mathrm{kg}$, y la cual ha demostrado ser eficiente en diversos tipos de muestras. Antes de cuantificar las muestras por cromatografía líquida de alto rendimiento, el contenido de OTA debe aislarse y extraerse. Si bien distintos métodos han sido utilizados para este fin, la extracción por cromatografía de inmunoafinidad es el método más difundido ya que ha demostrado mayor eficiencia. El método se basa en la propiedad que tienen ciertos anticuerpos para unirse específicamente a las moléculas de OTA mediante una reacción antígeno-anticuerpo. De esta forma, los anticuerpos presentes en la fase estacionaria de las columnas utilizadas en esta técnica pueden retener el contenido de OTA mientras dejan pasar los demás componentes de la muestra ${ }^{2,3}$.

Trabajos acerca de la presencia de OTA en cacao se han realizado en Nigeria, Costa de Marfil, Guinea, Camerún, Italia, Brasil, entre otros. En estos trabajos se ha podido concluir que los organismos generadores de toxina están presentes desde la cosecha, siendo la etapa de fermentación del cacao donde proliferan en mayor cantidad. De igual modo, se ha demostrado que el secado del cacao y su almacenamiento en lugares secos, siempre que se realicen bien, disminuyen en gran magnitud la presencia de $\mathrm{OTA}^{4}$.

En el Perú no hay estudios profundos acerca de la incidencia de OTA en cacao, aun cuando hay una necesidad de estos para fijar un límite máximo ${ }^{5}$. Asimismo, no se cuenta con una norma de los niveles permitidos de OTA para el cacao y sus derivados; por ello, en este trabajo se planteó el análisis de la presencia de esta toxina en granos del "cacao blanco de Piura", un tipo de cacao oriundo del Perú considerado de alta calidad. El análisis se realizó en las etapas de cosecha, fermentado, secado y almacenado del proceso de la producción del cacao, con el fin de determinar cómo la OTA se distribuye en la cáscara del grano, como en el cotiledón y detectar las etapas que requieren mayor cuidado durante el proceso. Este trabajo busca ser el primer paso de un estudio más profundo acerca de la incidencia de la Ocratoxina A en el cacao del Perú, así como de los métodos utilizados para su determinación.

\section{PARTE EXPERIMENTAL}

La investigación se realizó en el Instituto de Investigación en Ciencias Farmacéuticas y Recursos Naturales "Juan de Dios Guevara" de la Facultad de Farmacia y Bioquímica de la UNMSM. Laboratorio de Investigación y Certificaciones (LABICER) de la Universidad Nacional de Ingeniería. 


\section{Reactivos}

Bicarbonato de sodio $\left(\mathrm{NaHCO}_{3}\right)$ de grado analítico (Merck Peruana) 99\%, estándar de ocratoxina A (OTA) 98\% (Sigma Aldrich), metanol (MeOH) grado HPLC 99,9\% (Scharlau), ácido acético $\left(\mathrm{CH}_{3} \mathrm{COOH}\right)$ grado HPLC $98 \%$ (Merck Peruana), acetonitrilo $\left(\mathrm{CH}_{3} \mathrm{CN}\right)$ grado HPLC 99,85\% (Scharlau), buffer fosfato salino (PBS) en tabletas $\mathrm{pH}=7,40$ (Sigma Aldrich). El agua utilizada fue purificada en un equipo Elga Purelab CLASSIC UV.

\section{Equipos}

Cromatógrafo Líquido de Alto Rendimiento-SHIMADZU: desgasificador DGU-20A5R, automuestreador SIL-30AC, bomba cuaternaria LC-30AD, horno de columna: CTO-20AC, detector de fluorescencia RF-20Axs, control de sistema SBM-20A. Columna cromatográfica RP C18 de $250 \mathrm{~mm}$ x 4,6 mm ID x $5 \mu \mathrm{m}$ partícula (Restek). Balanza analítica digital SARTORIUS CPA225D, equipo ultrasonido WISD Laboratory Instruments, equipo de agua ultrapura ELGA PURELAB CLASSIC UV, columnas de inmunoafinidad para ocratoxina OCHRAPREP.

\section{Colecta de la muestra}

Los granos de Theobroma cacao L. "cacao blanco" fueron colectados del centro de acopio de la Asociación de Pequeños Productores de Cacao de Piura (APPROCAP) localizado en el distrito de San Juan de Bigote, provincia de Morropón, Región Piura, Perú. Las actividades de muestreo se realizaron a una temperatura de $37^{\circ} \mathrm{C}$ y $70 \%$ de humedad atmosférica durante el mes de marzo del 2016. Las muestras se colectaron en distintas etapas del proceso de la producción del cacao, consistiendo en la cosecha y apertura del fruto de cacao, fermentado en cajas de fermentación durante seis días, secado al sol por otros seis días y para su almacenamiento y posterior venta. Se seleccionaron cuatro tipos de muestra: cacao recién cosechado (M1), granos de cacao fermentados (M2), granos de cacao secados (M3) y granos de cacao almacenados en sacos (M4).

Las muestras M1 se colectaron en forma de fruto a diferencias de las demás que se colectaron en forma de grano.

\section{Pre-tratamiento de las muestras}

De las muestras M1 se extrajeron los granos de los frutos y se secaron en estufa a $40^{\circ} \mathrm{C}$ por dos días; de igual forma, se secaron las muestras M2 que habían sido colectadas húmedas. Las muestras M3 y M4 se secaron por un día en estufa a $40^{\circ} \mathrm{C}$, para eliminar la humedad que pudieron haber ganado durante su almacenamiento. Las muestras peladas, los cotiledones y las cáscaras se trataron en un mortero y luego en molino de cuchillas. Una vez trituradas se homogenizaron y se conservaron a $10^{\circ} \mathrm{C}$ para su análisis al día siguiente.

\section{Preparación de los estándares de ocratoxina $\mathrm{A}$}

La solución stock de OTA y los estándares se prepararon utilizando una solución de trabajo con el fin de reproducir el solvente de las muestras, que consistió en una mezcla de metanol: ácido acético: agua (49:1:50). Se utilizó esta mezcla de solventes para reproducir la condición inicial de las mezcla de eluyentes elegida en base a las recomendaciones dadas por Tafuri et 
al. $(2004)^{6}$. La solución stock del estándar de OTA se preparó mediante la disolución de $1 \mathrm{mg}$ de estándar puro en $100 \mathrm{~mL}$ del solvente de trabajo $(10 \mathrm{ppm})$, un mililitro de esta solución stock se disolvió en $100 \mathrm{~mL}$ de solución de trabajo con el fin de obtener el stock de trabajo (100 ppb); a partir de este stock de trabajo se prepararon los diferentes estándares utilizados en la construcción de la curva de calibración que fueron: 0,$25 ; 0,5 ; 1,0 ; 5,0$ y 10,0 ppm. El stock de trabajo fue preparado antes del análisis.

\section{Extracción y purificación de la ocratoxina A}

Este proceso se realizó por triplicado en cada uno de los cuatro grupos de muestra a analizar y, en cada una, se analizó tanto el cotiledón como la cáscara de la semilla. El método de acondicionamiento usado antes de colocar la muestra fue humedecer las columnas con agua grado HPLC y posteriormente con la solución PBS, de acuerdo con el método B descrito por Tafuri et al. $(2004)^{6}, 10 \mathrm{~g}$ de muestra a analizar se re-suspendieron en $200 \mathrm{~mL}$ de bicarbonato de sodio $1 \%$, se homogenizó por tres minutos y se filtró por papel filtro Whatman $\mathrm{N}^{\circ} 40 ; 20$ $\mathrm{mL}$ del filtrado se adhirieron a $20 \mathrm{~mL}$ de buffer PBS a pH: 7,4; los $40 \mathrm{~mL}$ de esta solución se purificaron en la columna de inmunoafinidad y se eluyeron a velocidad de 1-2 gotas por segundo. La columna se lavó con $20 \mathrm{~mL}$ de agua y se eluyó el contenido de OTA con 1,5 mL de metanol (ácido acético al 2\%) y $1,5 \mathrm{~mL}$ de agua.

\section{Cuantificación por cromatografía líquida de alto rendimiento}

Los análisis se llevaron a cabo mediante cromatografía líquida de alto rendimiento con detección por fluorescencia siguiendo los parámetros recomendados por Tafuri et al. (2004) .

La longitud de onda de excitación fue de $333 \mathrm{~nm}$ y la de emisión fue de $460 \mathrm{~nm}$. La temperatura del horno de la columna fue de $25{ }^{\circ} \mathrm{C}$. La elución fue a un flujo constante de

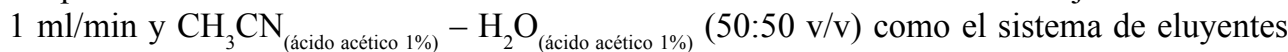
inicial. El sistema inicial fue linealmente modificado a un $\mathrm{CH}_{3} \mathrm{CN} 100 \%$ en 20 minutos. Después de 3 minutos de permanecer constante, el sistema fue restablecido linealmente a su condición inicial en 10 minutos, sumando un tiempo total de 33 minutos por análisis. Los eluyentes fueron preparados en el momento y filtrados $(0,45 \mu \mathrm{m})$ antes de su uso.

Para la lectura de los estándares se realizaron tres inyecciones y dos para el análisis de las muestras. Todas las muestras se filtraron a través de filtro de jeringa $(0,45 \mu \mathrm{m})$ antes de la inyección $(20 \mu \mathrm{L})$ a la columna cromatográfica.

La identificación de la OTA se realizó utilizando el tiempo promedio de retención de las diluciones de trabajo. Este tiempo se obtuvo midiendo nueve veces el estándar de trabajo a una concentración de $5 \mathrm{ppb}$, procedimiento que también fue útil para obtener el límite de detección (LDD). El tiempo de retención promedio fue de 8,387 (RSD=0,302\%) y el LDD fue de $0,194 \mathrm{ppb}(\mathrm{RSD}=4,978 \%$ ). En la figura 1 se muestra el cromatograma de una de las corridas del stock de trabajo de 5 ppb. 


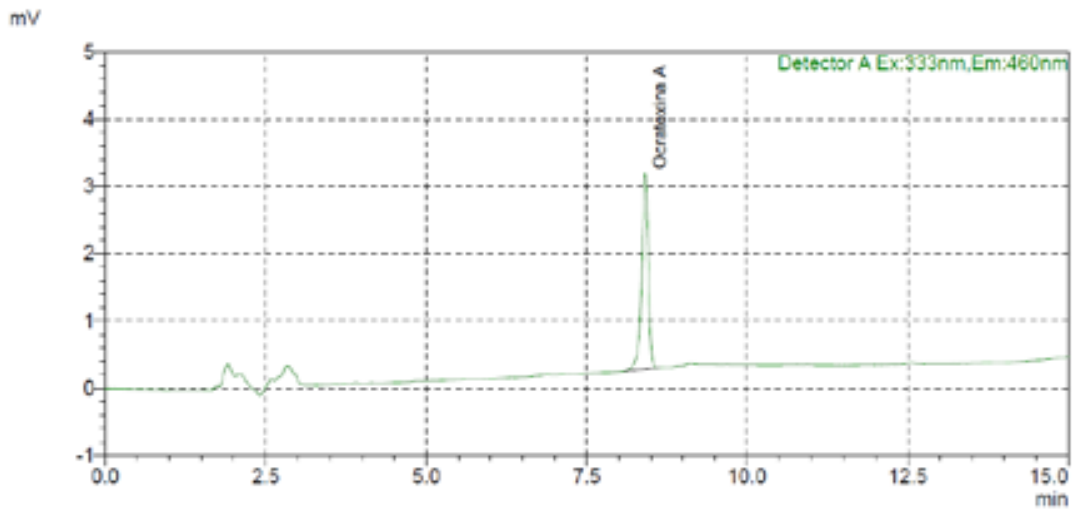

Figura 1. Cromatograma de una de las repeticiones del estándar de trabajo (5 ppb).

El tiempo de retención fue de 8,412 s.

La cuantificación de la OTA se realizó comparando el área de los picos de las muestras analizadas y de los estándares de trabajo. En la figura 2 se observa la curva de calibración para 0,$25 ; 0,5 ; 1,0 ; 5,0$ y 10,0 ppm la cual fue descrita en la preparación de los estándares de ocratoxina.

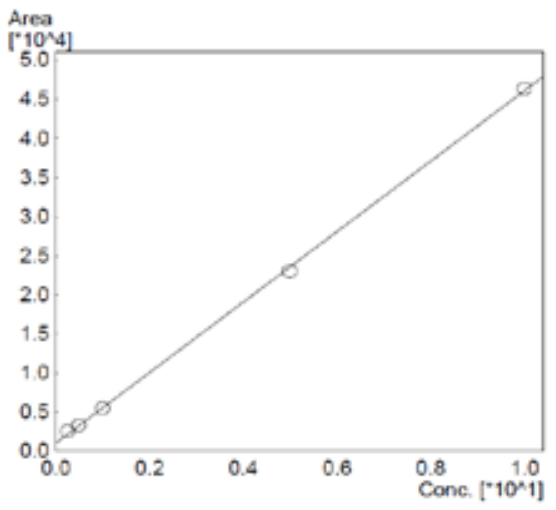

Figura 2. Curva de calibración utilizada para la cuantificación de la OTA.

\section{RESULTADOS Y DISCUSIÓN}

En este trabajo se analizó el contenido de OTA en los procesos de cosecha (M1), fermentado (M2), secado (M3) y almacenado (M4) de cacao, tanto en la cáscara como en el cotiledón. De estos procesos, solo las muestras M2 presentaron niveles detectables de OTA. En la tabla 1 se muestran los resultados obtenidos en $\mu \mathrm{g}$ de OTA $/ \mathrm{kg}$ de cacao con sus respectivas desviaciones estándar tal cual fueron calculadas utilizando el Excel Office 2010. 
Tabla 1. Resultados de la cuantificación de OTA en las diferentes muestras.

\begin{tabular}{rcc}
\hline Muestra & Proceso & Concentración $(\mu \mathrm{g} / \mathrm{kg})$ \\
M1 cotiledón & cosecha & $<$ L.D.D \\
M1 cáscara & cosecha & $<$ L.D.D \\
M2 cotiledón & fermentado & $1.92 \pm 0.02$ \\
M2 cáscara & fermentado & $<$ L.D.D \\
M3 cotiledón & secado & $<$ L.D.D \\
M3 cáscara & secado & $<$ L.D.D \\
M4 cotiledón & almacenado & $<$ L.D.D \\
M4 cáscara & Almacenado & $<$ L.D.D \\
\hline
\end{tabular}

L.D.D= Límite de detección.

El valor obtenido para la muestra M2 (cotiledón) es mayor que el límite sugerido por el gobierno de Italia $(0,5 \mu \mathrm{g} / \mathrm{kg})$, el cual es el único miembro de la Unión Europea que actualmente clama por el establecimiento de un límite legal para el cacao y derivados. Hasta la fecha, aún sigue en debate el establecimiento de un límite legal por parte del gobierno norteamericano. De igual forma, el Perú tampoco cuenta con un límite legal establecido.

El cromatograma de la muestra que presento OTA (M2 cotiledón) se presenta en la figura 3. El cromatograma de una de las muestras en las cuales no se detectó OTA (M1 cotiledón) se muestra en la figura 4.

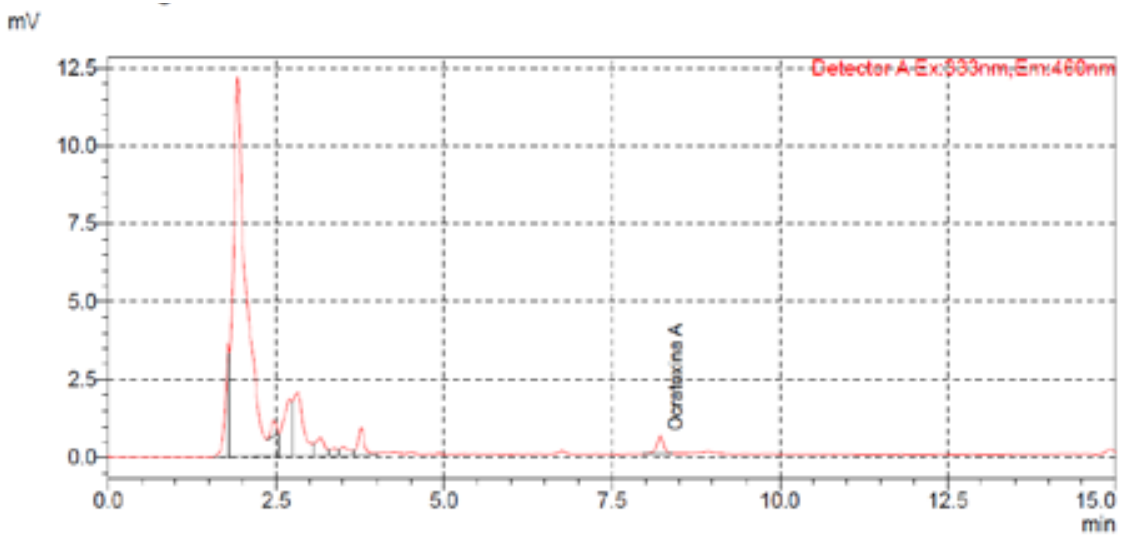

Figura 3. Cromatograma de la muestra M2 - cotiledón. 


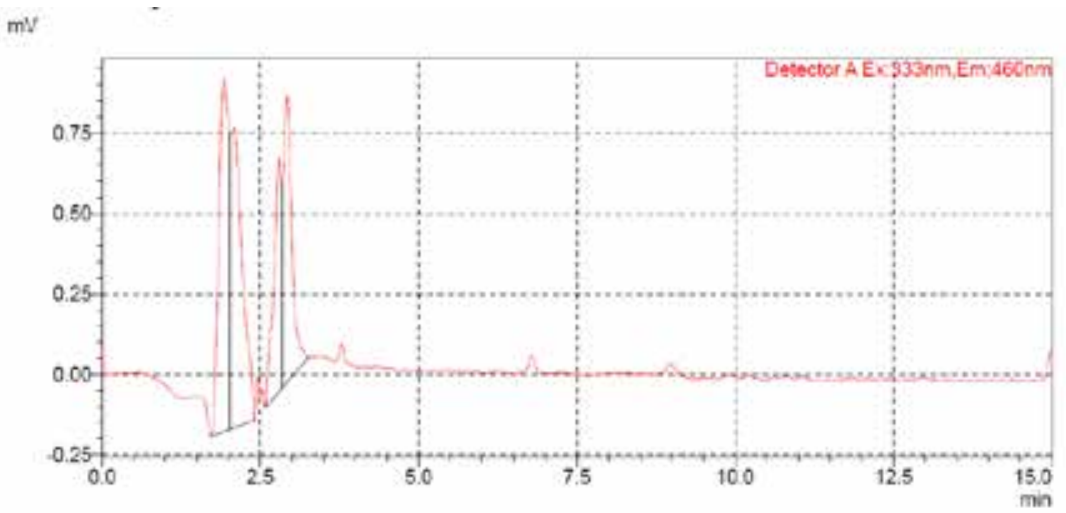

Figura 4. Cromatograma de la muestra M1 - cotiledón.

Los resultados obtenidos indican que el tratamiento de granos de cacao muestra cierta deficiencia en la etapa del fermentado. Estos resultados se corresponden con los encontrados anteriormente en otras investigaciones, las cuales han demostrado que la mayor concentración de hongos productores de OTA (principalmente del género Aspergillus) se encuentra en la etapa final de la fermentación ${ }^{7}$.

La ausencia de OTA en los granos sin abrir se debe a que los hongos generadores de dicha toxina no penetran en el fruto, a menos que este haya sufrido daños físicos o infectado por otros agentes patógenos, caso en el cual se han reportado altas concentraciones de OTA $^{7}$.

Una vez que los granos se abren, la presencia de diversos microorganismo comienza inmediatamente. Estos microorganismos provienen de fuentes como el medio ambiente, la mano de los agricultores, los instrumentos que se utilizan para cortar los frutos de cacao, entre otros ${ }^{2}$. Los primeros microorganismos en presentarse en los granos son las levaduras fermentativas, pero luego son superadas por las bacterias generadoras de ácido acético y ácido láctico $\mathrm{y}$, ocasionalmente, los hongos filamentosos generadores de toxinas. La proliferación de estos hongos se ve favorecida por el alto contenido de humedad de los granos (55\%), la cual penetra en ellos y los hincha. Este hinchamiento también reduce la acidez de los granos que es un factor inhibidor del crecimiento de los hongos filamentosos. Este hinchamiento interno que permite la penetración de los hongos explica por qué en esta etapa la OTA se encontró en la parte interna del grano ${ }^{8}$.

El haber encontrado OTA en solo una de las tres muestras extraídas en la etapa de fermentación, demuestra lo heterogéneo de la forma en que la OTA se reparte. ${ }^{5}$ Copetti et $a l^{9}$ analizaron la presencia de OTA en muestras de cacao de Brasil y encontró que menos del $5 \%$ del cacao fermentado estaba infectado y que este cacao se encontraba en su gran mayoría en la superficie; por lo que los resultados encontrados en el cacao de Piura evidencian la necesidad de mejorar la forma en que los cajones de fermentación se protegen del medio ambiente. Cabe mencionar que otro factor que influencia la presencia de OTA, en el cacao, es la pulpa en los cajones ya que una mayor cantidad de ella ayuda a crear una barrera física contra la penetración de los microorganismos productores de toxinas ${ }^{10}$. 
La cantidad de hongos generadores de OTA es menor en la etapa del secado, debido a que este proceso disminuye la actividad acuosa y la humedad desde 55 hasta $7 \%{ }^{8}$. Con el fin de prevenir la presencia de microorganismos productores de toxinas se recomienda el secado directo al sol, ya que la radiación directa afecta la producción de OTA por parte de los microorganismos ${ }^{4}$. Otro factor que explica que no se haya detectado OTA, en esta etapa de la producción, es el hecho de que los agricultores del centro de acopio de APPROCAP retiran los granos que no presentan buen estado. Esta práctica ayuda a evitar la posible contaminación cruzada entre granos.

Es sabido que otra etapa crítica en la producción de OTA es el almacenaje. La cáscara del grano de cacao es higroscópica por lo que los granos deben almacenarse protegidos de la humedad ${ }^{4}$. En el caso del centro de acopio de APPROCAP, los granos de cacao de diferentes orígenes se almacenan en sacos de yute en forma separada y sobre parihuelas dejando poco aire en el interior de los sacos. Tal como indican los resultados, esta práctica es exitosa en su objetivo de evitar la proliferación de la toxina.

Los resultados obtenidos evidencian la necesidad de mejorar la forma en que las cajas de fermentación sean protegidas del medio ambiente en el centro de acopio de APPROCAP. Estos resultados se corresponden con estudios anteriores que demuestran que es en esta etapa donde principalmente proliferan los hongos productores de toxina. Las otras etapas de producción de granos de cacao: cosecha, secado y almacenado, no mostraron concentraciones de OTA detectables. Con el fin profundizar en los resultados obtenidos por este estudio se recomienda continuar con los análisis en otras temporadas de cosecha, además de utilizar nuevos métodos de cuantificación y compararlos.

\section{CONCLUSIONES}

La cuantificación de OTA por cromatografía líquida de alto rendimiento y extracción por cromatografía de inmunoafinidad es un método eficiente, el cual es adecuado para el análisis de diversas matrices como en el caso del cacao. Los resultados obtenidos indican que el cacao blanco de Piura presenta OTA en la etapa de fermentación, la más proclive a sufrir dicha contaminación. El contenido de OTA encontrado en la etapa de fermentado fue de 1,92 $\pm 0,02$ $\mu \mathrm{g} / \mathrm{kg}$. Las etapas de cosecha, secado y almacenamiento del proceso de producción de cacao en Piura no mostraron cantidades detectables de OTA.

\section{AGRADECIMIENTOS}

A la Asociación de Pequeños Productores de Cacao de Piura (APPROCAP), al Laboratorio de Investigación y Certificaciones (LABICER) de la Facultad de Ciencias de la Universidad Nacional de Ingeniería y al Instituto de Investigación en Ciencias Farmacéuticas y Recursos Naturales “Juan de Dios Guevara” de la Facultad de Farmacia y Bioquímica de la Universidad Nacional Mayor de San Marcos. 


\section{REFERENCIAS BIBLIOGRÁFICAS}

1. Malir F, Ostry V, Pfohl-Leszkowicz A, Novotna E. Ochratoxin A: Developmental and Reproductive Toxicity-An Overview. Birth Defects Res B. 2013;98(6):493-502.

2. Sugita-konishi Y, Tanaka T, Nakajima M, Fujita K, Norizuki H, Mochizuki N, et al. The comparison of two clean-up procedures, multifunctional column and immunoaffinity column, for HPLC determination of ochratoxin A in cereals, raisins and green coffee beans. Talanta. 2006;69(3):650-5.

3. Afsah-Hejri L, Jinap S, Mirhosseini H. Ochratoxin A quantification: Newly developed HPLC conditions. Food Control. 2012;23(1):113-9.

4. Copetti M, Iamanaka B, Pitt J, Taniwaki M. Fungi and mycotoxins in cocoa: From farm to chocolate. Int J Food Microbiol. 2014;178:13-20.

5. Chire G, Valdivia R, Ureña M. Ocratoxina A en cacao y derivados. Medidas preventivas. Ciencia e Investigación, 2014;17(1):9-15.

6. Tafuri A, Ferracane R, Ritieni A. Ochratoxin A in Italian marketed cocoa products. Food Chem. 2004;88(4):487-94.

7. Mounjouenpou P, Gueule D, Fontana-Tachon A, Guyot B, Tondje P, Guiraud J. Filamentous fungi producing ochratoxin a during cocoa processing in Cameroon. Int $\mathbf{J}$ Food Microbiol. 2008;121(2):234-41.

8. De Magalhães J, Sodré G, Viscogliosi H, Grenier-Loustalot M. Occurrence of Ochratoxin A in Brazilian cocoa beans. Food Control. 2011;22(5):744-8.

9. Copetti M, Pereira J, Iamanaka B, Pitt J, Taniwaki M. Ochratoxigenic fungi and ochratoxin A in cocoa during farm processing. Int J Food Microbiol. 2010;143(1-2):6770.

10. Copetti M, Iamanaka B, Mororó R, Pereira J, Frisvad J, Taniwaki M. The effect of cocoa fermentation and weak organic acids on growth and ochratoxin A production by Aspergillus species. Int J Food Microbiol. 2012;155(3):158-64. 